\title{
Biofilm production using distinct media and antimicrobial susceptibility profile of Pseudomonas aeruginosa
}

\begin{abstract}
Biofilm production is an important mechanism for bacterial survival and its occurrence together with antimicrobial resistance represents a challenge for clinical management. Here, we evaluated the ability for biofilm production among $P$. aeruginosa isolates from patients with or without cystic fibrosis (CF) using two distinct media, besides determining the antimicrobial susceptibility profile of these isolates for eight antimicrobial agents. The ability for biofilm production when TSB medium was used was higher than when used CF sputum media $(\mathrm{p}=0.0198)$. However, P. aeruginosa isolates from CF have demonstrated similar performance for biofilm production, independently of the medium used. Besides, among the biofilm-producing isolates, those recovered from CF were more resistant to the carbapenems (meropenem and imipenem) agents than those isolates from non-CF isolates.
\end{abstract}

Keywords: biofilm; Pseudomonas aeruginosa; cystic fibrosis; antimicrobial resistance.

\section{INTRODUCTION}

Pseudomonas aeruginosa is one of the leading causes of nosocomial infections and it has a crucial role in cystic fibrosis (CF), being responsible for high level mortality and morbidity. ${ }^{1,2}$ The lung of CF patients is characterized by high mucus production, which blocks clearance mechanisms. P. aeruginosa grows in CF lung as microcolonies promoting resistance to immunologic cells defense as well as persistence character in face of nutritional deficiency and oxygen deprivation. ${ }^{3,4}$

It is well recognized that biofilm mode of growth can promote resistance to antimicrobial agents, and its occurrence in the infection process has been considered a limiting factor for therapeutic success. ${ }^{5,6}$ Moreover, there are just a few studies that evaluate biofilm production on mucus and sputum in vitro and under these conditions one could expect better correlation in vivo production.

The aim of the present study was to compare biofilm production among $P$. aeruginosa isolates using tryptic soy broth (TSB), as conventional medium, and a pool of $\mathrm{CF}$ sputum, as alternative medium in microti- ter plate assay. Besides, it was also compared the antimicrobial susceptibility profile of $P$. aeruginosa biofilm-producing isolates recovered from $\mathrm{CF}$ and non-CF patients against eight antimicrobial agents, using TSB medium.

\section{MATERIAL AND METHODS}

\section{Samples and media}

A total of 124 P. aeruginosa isolates, 52 from CF $(\mathrm{PCF}+)$ and 72 from non-CF (PCF-) patients, recovered form sputum and endotracheal aspirates were evaluated.

Sputum specimens from randomly selected CF patients were collected into a sterile bottle to prepare a pool of sputum. To certify the quality of the sputum, an amount of each specimen was stained by Gram and microscopically inspected (100x) using the presence of $<10$ epithelial cells per field as criterion. After, this pool was homogenized mechanically, autoclaved at $121^{\circ} \mathrm{C}$ during $15 \mathrm{~min}$ and stored at $-20^{\circ} \mathrm{C}$.

\section{Biofilm formation}

Quantitative determination of biofilm was made using a microtiter plate assay in accordance with O'Toole et al., ${ }^{7}$ with some modifi-
Authors

Leandro Reus Rodrigues Perez ${ }^{1}$

Afonso Luís Barth ${ }^{2}$

${ }^{1}$ Microbiology Unit, Hospital Mãe de Deus; Post-graduation Program in Pharmaceutical Sciences, Universidade Federal do Rio Grande do Sul (UFRGS), Porto Alegre, RS, Brazil

${ }^{2}$ Post-graduation Program in Pharmaceutical Sciences, UFRGS; Microbiology Unit and Molecular Biology Service, Hospital de Clínicas, Porto Alegre, RS, Brazil

Submitted on: 03/05/2010 Approved on: 03/20/2010

Correspondence to: Leandro Reus Rodrigues Perez

Laboratory of Clinical Analysis, Faculty of Pharmacy, UFRGS, Av. Ipiranga, 2752/303 90610-000,

Porto Alegre, RS

Brazil

Phone: +55 (51) 33085412

Fax: +55 (51) 33085437

leandro.reus@gmail.com

We declare no conflict of interest.

(C)2011 Elsevier Editora Ltda. All rights reserved. 
cations. In brief, three to five colonies were suspended in 5 $\mathrm{mL}$ of TSB and incubated overnight without shaking. After incubation, the stationary-phase culture was vortexed and thereafter diluted $1: 100$ in TSB with $0.25 \%$ glucose, and $200 \mu \mathrm{L}$ of this solution was incubated in 96-well plates overnight at $35 \pm 1^{\circ} \mathrm{C}$. Media with suspended bacteria was then removed; the plates were carefully washed four times with water and air-dried before $200 \mu \mathrm{L}$ of $0.9 \%$ crystal violet solution (Sigma, Stockholm, Sweden) was added for 15 min. After removing the dye solution and washing with water, the attached dye was solubilized with $95 \%$ ethanol and the optical density of the adherent biofilm was determined dual with $450 / 630 \mathrm{~nm}$ microtiter plate reader (OrthoReader II, Ortho diagnostic systems, New Jersey, USA). In the experiments, we have just used TSB with $0.25 \%$ glucose as a negative control (background absorbance). All isolates were tested at least three times in triplicate. For interpretation of the results, the isolates were classified as follow: non-producing, weak, moderate, and strong-producing, based on the following optic density (OD) average values:

$\mathrm{OD}$ (isolate) $\leq \mathrm{OD}$ (control) = non-biofilm-producing;

$\mathrm{OD}$ (control) $\leq \mathrm{OD}$ (isolate) $\leq 2 \mathrm{OD}$ (control) $=$ weakproducing;

$2 \mathrm{OD}($ control $) \leq \mathrm{OD}($ isolate $) \leq 4 \mathrm{OD}($ control $)=$ moder ate-producing;

$4 \mathrm{OD}($ control $) \leq \mathrm{OD}$ (isolate $)=$ strong-producing. ${ }^{8}$

\section{Control strains}

A reference biofilm-producing strain of Pseudomonas aeruginosa $\mathrm{PAO} 1$ and a non-producing strain of Pseudomonas aeruginosa ATCC 27853 were used as controls.

\section{Antimicrobial susceptibility profile}

Susceptibility profile to amikacin, aztreonam, cefepime, ceftazidime, ciprofloxacin, gentamicin, imipenem, meropenem and piperacillin-tazobactam was assessed by the
VITEK automated system (bioMérieux, Marcy l'Étoile, France) using GNS-655 cards (bioMérieux, Marcy l'Étoile, France) in accordance with manufacturer's instructions and breakpoints established by CLSI documents. ${ }^{9}$ The antimicrobial susceptibility profile of the biofilm producers was evaluated based on the TSB results.

\section{Statistical analysis}

Fisher's exact test was used to compare biofilm production by TSB and CF sputum media, as well as biofilm production of cystic fibrosis and non-cystic fibrosis patients in sputum media. A p-value $<0.05$ was considered statistically significant.

\section{RESULTS}

\section{Biofilm production}

In TSB, 96.1\% (50/52) of the PCF+ isolates have produced biofilm. Among PCF- isolates, 91.7\% (66/72) have produced biofilm. A strong capacity to form biofilm has been observed in $56 \%(28 / 50)$ and in $39.4 \%(26 / 66)$ of the $\mathrm{PCF}+$ and PCF- isolates, respectively. Using CF sputum, 92.3\% (48/52) of the PCF+ isolates showed to be biofilmproducers and most of these were classified as moderate biofilm-producers (58.3\%). Among PCF- isolates, $66.7 \%(48 / 72)$ were biofilm-producers and $46 \%(22 / 48)$ of these isolates were classified as strong biofilm-producers (Table 1). The difference in the ability of producing biofilm between PCF- and PCF+ isolates, $66.7 \%$ vs $92.3 \%$, respectively, showed to be extremely significant $(\mathrm{p}=0.0009)$.

Of the $52 \mathrm{PCF}+$ isolates that were evaluated using $\mathrm{CF}$ sputum, only two were unable to produce biofilm. However, our results showed that biofilm production using TSB was higher than when used CF sputum $(\mathrm{p}=0.0198)$ (Table 1$)$.

Table 1. Biofilm production among $\mathrm{P}$. aeruginosa isolates using two distinct media

\begin{tabular}{|c|c|c|c|c|c|c|c|c|}
\hline \multirow{2}{*}{$\begin{array}{l}\text { Source of the } \\
\text { isolates }\left(n^{o}\right)\end{array}$} & \multicolumn{4}{|c|}{ Biofilm production in TS } & \multicolumn{4}{|c|}{ Biofilm production in CF sputum } \\
\hline & Non producer & Weak & Moderate & Strong & Non producer & Weak & Moderate & Strong \\
\hline $\mathrm{PCF}+{ }^{\mathrm{a}}(52)$ & 2 & 6 & 16 & 28 & 4 & 8 & 28 & 12 \\
\hline PCF-b $(72)$ & 6 & 14 & 26 & 26 & 24 & 12 & 14 & 22 \\
\hline Subtotal (124) & 8 & 20 & 42 & 54 & 28 & 20 & 42 & 34 \\
\hline Total (124) & $8 *$ & & $116^{*}$ & & $28 *$ & & $96^{*}$ & \\
\hline
\end{tabular}

aPCF+, Pseudomonas aeruginosa from cystic fibrosis patients.

bPCF-, Pseudomonas aeruginosa from non-cystic fibrosis patients.

* Biofilm production considering TSB and CF sputum were statistically significant $(\mathrm{p}=0.0198)$. 
Table 2. Antimicrobial susceptibility profile of biofilm producers of cystic fibrosis patients vs. non-cystic fibrosis patients using TSB medium ${ }^{a}$

\begin{tabular}{|c|c|c|c|c|c|c|}
\hline \multirow{2}{*}{$\begin{array}{l}\text { Antimicrobial } \\
\text { agents }\end{array}$} & \multicolumn{3}{|c|}{ Cystic fibrosis patients $\left(\mathrm{n}^{\mathrm{b}}=50\right)$} & \multicolumn{3}{|c|}{ Non-cystic fibrosis patients $\left(n^{b}=66\right)$} \\
\hline & \% susceptible & $\%$ intermediate $^{c}$ & $\%$ resistant $^{\mathrm{c}}$ & \% susceptible & $\%$ intermediate $^{c}$ & $\%$ resistant \\
\hline Amikacin & 76 & 8 & 16 & 72.7 & - & 27.3 \\
\hline Aztreonam & 52 & 16 & 32 & 57.7 & 9 & 33.3 \\
\hline Cefepime & 28 & 40 & 32 & 30.3 & 24.2 & 45.5 \\
\hline Ceftazidime & 40 & 20 & 40 & 30.3 & 24.2 & 45.5 \\
\hline Ciprofloxacin & 52 & - & 48 & 54.5 & - & 45.5 \\
\hline Gentamicin & 48 & 28 & 24 & 63.6 & - & 36.4 \\
\hline Imipenem & 72 & 8 & 20 & 33.3 & 18.2 & 48.5 \\
\hline Meropenem & 92 & - & 8 & 36.4 & - & 63.6 \\
\hline Piperacillin-tazobactam & 68 & - & 32 & 81.8 & - & 18.2 \\
\hline
\end{tabular}

${ }^{a}$ Considering any degree of biofilm production (weak, moderate or strong).

${ }^{b} \mathrm{n}$ is the total number of isolates with biofilm producer status.

'Susceptible, intermediate and resistant phenotypes, from CLSI breakpoints.

\section{Antimicrobial susceptibility profile and biofilm production}

Table 2 illustrates the antimicrobial susceptibility profile of $P$. aeruginosa biofilm-producing from PCF+ and PCF-. Isolates with any degree of biofilm production (weak, moderate or strong) were considered as producer. Ciprofloxacin was the least active antimicrobial agent against the panel of PCF+ isolates (48\% resistant) followed by ceftazidime $(40 \%)$ and aztreonam, cefepime and piperacillin-tazobactam (32\%). A high proportion of PCF+ isolates displayed intermediate susceptibility to cefepime $(40 \%)$. On the other hand, isolates from PCF- were more susceptible to piperacillin-tazobactam (81.8\%) and more resistant to meropenem (63.6\%). Eighteen PCFisolates (25\%) were susceptible to all eight antimicrobial agents tested. Interestingly, only six PCF+ isolates (11.5\%) were susceptible to all eight antimicrobial agents tested.

\section{DISCUSSION}

The biofilm mode of growth is an important bacterial survival strategy. Pseudomonas aeruginosa can produce biofilm in lungs of chronically infected cystic fibrosis patients, protecting the bacteria against antimicrobial agents and immune responses. ${ }^{10,11}$ Biofilm starts growing on a surface involving a complex mechanism of interaction between bacteria and environment. This interaction, usually started by a primary adhesion, is reversible during the first couple of hours; later the adhesion becomes irreversible due to pili and polysaccharides of the bacteria. Bacterial adherence to the airway components, such as epithelial cells and mucus, has been considered to be a first step for colonization and sub- sequent establishment of an infection process. ${ }^{6}$ This mechanism is still poorly understood and a lot of possibilities such as impermeability of the matrix and bacterial persistent cells probably contribute for this dynamic status. Also, biofilm production has been related to the occurrence of genetic events such as hypermutation, transfer of genes and activation of distinct metabolic pathways. ${ }^{5}$ It is described that $P$. aeruginosa can interact with elements like mucins, lecithin, eukaryotic DNA, salt, iron and other elements present in the mucus and contribute for the biofilm production. ${ }^{6}$

In this study, we have evaluated the use of two media as vehicle to perform the test for biofilm production. Using a pool of CF sputum, isolates from $48 \mathrm{PCF}+$ and 48 PCF- showed to be biofilm producing, while isolates from $4 \mathrm{PCF}+$ and $24 \mathrm{PCF}$ - showed to be non-biofilm producing. Still, biofilm production in TSB was higher than in CF sputum ( $\mathrm{p}=0.0198)$. This can be explained by $P$. aeruginosa growing as microcolonies surrounded by matrix, mainly alginate, on sputum elements; in this condition is not observed the full adherence to the microplate well. On the other hand, the sputum can be a less rich medium than TSB to support biofilm production in both groups. But, interestingly, we have observed that $\mathrm{PCF}+$ isolates maintained their ability of biofilm production in both media. It is known that nutritionally rich conditions may be present in the CF airways, mainly amino acids. This is the main fact that promotes auxotrophic isolates. Besides, in the secretions of these patients substances that freely circulate in the airway environment are generated, especially in chronic processes. This condition may promote a selection of $P$. aeruginosa isolates with higher capacity for biofilm production. ${ }^{3}$ 
In this study, isolates with higher capacity to produce biofilm tended to have higher rates of resistance to the panel of antimicrobial agents tested, ranging from $86.6 \%$ (aztreonam) to $100 \%$ (amikacin) (data not shown). Delissalde and Amábile-Cuevas ${ }^{12}$ have reported in their study a rate slightly higher against six antimicrobials, ranging from $62 \%$ to $92 \%$ of biofilm production among resistant isolates after $24 \mathrm{~h}$ of incubation. The authors stated that multiresistant isolates had a higher capacity to produce biofilm both at 8 and $24 \mathrm{~h}$ of incubation. Interestingly, in this study the minority of $P$. aeruginosa isolates, not including isolates from $\mathrm{CF}$, was capable of producing biofilm. ${ }^{12}$ In our study, of the 72 isolates from PCF-, six (8.3\%) were unable to produce biofilm in TSB. Among isolates from PCF+, only two isolates (3.8\%) were unable to produce biofilm when we used TSB as medium. On the other hand, 24 PCF-isolates were unable to produce biofilm when CF sputum was used as medium. Four isolates from $\mathrm{PCF}+$ were unable to produce biofilm in CF sputum medium. Surprisingly, these four isolates were strong biofilm producers in TSB medium.

Knowledge of the susceptibility of $P$. aeruginosa to antimicrobial agents used for treatment not only serves as a guide to treatment for individual CF patients, but it is also valuable for identifying the prevalence of resistant strains within and among different treatment centers, given the mobility of some patients. Our results have demonstrated that isolates from PCF+ and PCF- possess distinct profiles against a panel of antimicrobials. In particular, very high levels of resistance to two main drugs (meropenem and imipenem) were evident among isolates from PCF-, casting doubt on the adequacy of their empirical use in this patient group. This difference between the susceptibility profiles can be explained by the nature of the infection, since isolates from PCF- were recovered from patients admitted in the intensive care unit, nosocomial setting with a great selective pressure for extended spectrum antimicrobials such as carbapenems. In fact, our results showed that isolates from PCF- displayed more resistance to carbapenems than isolates from PCF+. Surprisingly, susceptibility to all eight antimicrobial agents was higher among isolates from PCF- than isolates from $\mathrm{PCF}+(25 \%$ vs $11.5 \%)$.

Invariably, these isolates were able to produce biofilm in distinct media. Although biofilm production has recently been studied more deeply, more studies are still needed to improve our understanding about the link between virulence factors and antimicrobial resistance.

We are aware of the fact that CF sputum medium used here is not entirely representative of CF lung environment because other active compounds and growth conditions, including anaerobic atmosphere, may be also present. ${ }^{4,6}$ On the other hand, P. aeruginosa isolates from PCF+ apparently displayed, under distinct conditions, the same feature for the biofilm production.
Biofilm production and antimicrobial resistance are among the main bacterial defense apparatus during an infectious process, and both represent currently a great challenge to the therapeutic practice.

\section{ACKNOWLEDGEMENTS}

We are grateful to Karen Oliveira Silva for the support with microbiological tests and Cícero Dias for critical analysis.

\section{REFERENCES}

1. Emerson J, Rosenfeld M, McNamara S, Ramsey B, Gibson RL. Pseudomonas aeruginosa and other predictors of mortality and morbidity in young children with cystic fibrosis. Pediatr Pulmonol 2002; 34:91-100.

2. Kosorok MR, Zeng L, West SE et al. Acceleration of lung disease in children with cystic fibrosis after Pseudomonas aeruginosa acquisition. Pediatr Pulmonol 2001; 32:277-87.

3. Sriramulu DD, Lünsdorf H, Lam JS, Römling U. Microcolony formation: a novel model of Pseudomonas aeruginosa for the cystic fibrosis lung. J Med Microbiol 2005; 54:667-76.

4. Jesaitis AJ, Franklin MJ, Berglund D et al. Compromised host defense on Pseudomonas aeruginosa biofilms: characterization of neutrophil and biofilm interactions. J Immunol 2003; 171:4329-39.

5. Singh R, Paul D, Jain RK. Biofilms: implication in bioremediation. Trends Microbiol 2006; 14:389-97.

6. Worlitzsch D, Tarran R, Ulrich M et al. Effects of reduced mucus oxygen concentration in airway Pseudomonas infections of cystic fibrosis patients. J Clin Invest 2002; 109:317-25.

7. O'Toole GA, Pratt LA, Watnick PI, Newman DK, Weaver VB, Kolter R. Genetic approaches to study biofilms. Methods Enzymol 1999; 310:91-109.

8. Stepanovic S, Vucovic D, Hola V et al. Quantification of biofilm in microtiter plates: overview of testing conditions and practical recommendations for assessment of biofilm production by staphylococci. APMIS 2007; 115:891-9.

9. Clinical and Laboratory Standards Institute - CLSI. Performance standards for antimicrobial susceptibility testing. 19th informational supplement M100-S19. Wayne, PA: CLSI/(NCCLS), 2009

10. Costerton JW, Stewart PS, Greenberg EP. Bacterial biofilms: a common cause of persistent infections. Science 1999; 284:1318-22.

11. Frederiksen B, Lanng S, Koch C, Hoiby N. Improved survival in the Danish center-treated cystic fibrosis patients: results of aggressive treatment. Pediatr Pulmonol 1996; 21:153-58.

12. Delissalde F, Amábile-Cuevas CF. Comparison of antibiotic susceptibility and plasmid content, between biofilm producing and non-producing clinical isolates of Pseudomonas aeruginosa. Int J Antimicrob Agents 2004; 24:405-8. 